\title{
Pretherapeutic resting-state fMRI profiles are associated with MR signature volumes after stereotactic radiosurgical thalamotomy for essential tremor
}

\author{
${ }^{*}$ Constantin Tuleasca, MD, PhD,,-4 Jean Régis, MD, ${ }^{5}$ Elena Najdenovska, PhD, ${ }^{2}$ \\ Tatiana Witjas, MD, ${ }^{6}$ Nadine Girard, MD, PhD, ${ }^{7}$ Thomas Bolton, ${ }^{8}$ Francois Delaire, ${ }^{5}$ \\ Marion Vincent, ${ }^{5}$ Mohamed Faouzi, $\mathrm{PhD},{ }^{9}$ Jean-Philippe Thiran, $\mathrm{PhD}, 3,4,10$ \\ Meritxell Bach Cuadra, PhD, ${ }^{2,3}$ Marc Levivier, MD, PhD, IFAANS, ${ }^{1,4}$ and Dimitri Van de Ville, PhD ${ }^{8,11}$ \\ ${ }^{1}$ Neurosurgery Service and Gamma Knife Center, ${ }^{2}$ Medical Image Analysis Laboratory (MIAL) and Department of Radiology, \\ Centre d'Imagerie BioMédicale (CIBM), and ${ }^{10}$ Department of Radiology, Centre Hospitalier Universitaire Vaudois (CHUV), \\ Lausanne, Switzerland; ${ }^{3}$ Signal Processing Laboratory (LTS 5), Ecole Polytechnique Fédérale de Lausanne (EPFL), Switzerland; \\ ${ }^{4}$ Faculty of Biology and Medicine, University of Lausanne, Switzerland; ${ }^{5}$ Stereotactic and Functional Neurosurgery Service \\ and Gamma Knife Unit, and ${ }^{6}$ Neurology Department, CHU Timone, Marseille, France; ${ }^{7}$ AMU, CRMBM UMR CNRS 7339, \\ Faculté de Médecine et APHM, Hôpital Timone, Department of Diagnostic and Interventional Neuroradiology, Marseille, France; \\ ${ }^{8}$ Medical Image Processing Laboratory, Ecole Polytechnique Fédérale de Lausanne (EPFL), Switzerland; ${ }^{9}$ nstitute of Social and \\ Preventive Medicine, Lausanne, Switzerland; and ${ }^{11}$ University of Geneva, Faculty of Medicine, Geneva, Switzerland
}

\begin{abstract}
OBJECTIVE Essential tremor (ET) is the most common movement disorder. Drug-resistant ET can benefit from standard stereotactic deep brain stimulation or radiofrequency thalamotomy or, alternatively, minimally invasive techniques, including stereotactic radiosurgery (SRS) and high-intensity focused ultrasound, at the level of the ventral intermediate nucleus (Vim). The aim of the present study was to evaluate potential correlations between pretherapeutic interconnectivity (IC), as depicted on resting-state functional MRI (rs-fMRI), and MR signature volume at 1 year after Vim SRS for tremor, to be able to potentially identify hypo- and hyperresponders based only on pretherapeutic neuroimaging data.

METHODS Seventeen consecutive patients with ET were included, who benefitted from left unilateral SRS thalamotomy (SRS-T) between September 2014 and August 2015. Standard tremor assessment and rs-fMRI were acquired pretherapeutically and 1 year after SRS-T. A healthy control group was also included $(n=12)$. Group-level independent component analysis (ICA; only $n=17$ for pretherapeutic rs-fMRI) was applied. The mean MR signature volume was 0.125 $\mathrm{ml}$ (median $0.063 \mathrm{ml}$, range $0.002-0.600 \mathrm{ml}$ ). The authors correlated baseline IC with 1-year MR signatures within all networks. A 2-sample t-test at the level of each component was first performed in two groups: group 1 ( $n=8$, volume < $0.063 \mathrm{ml}$ ) and group $2(\mathrm{n}=9$, volume $\geq 0.063 \mathrm{ml}$ ). These groups did not statistically differ by age, duration of symptoms, baseline ADL score, ADL point decrease at 1 year, time to tremor arrest, or baseline tremor score on the treated hand (TSTH; $p>0.05$ ). An ANOVA was then performed on each component, using individual subject-level maps and continuous values of 1-year MR signatures, correlated with pretherapeutic IC.

RESULTS Using 2-sample t-tests, two networks were found to be statistically significant: network 3, including the brainstem, motor cerebellum, bilateral thalamus, and left supplementary motor area $(S M A)\left(p_{F W E}=0.004\right.$, cluster size $\left.=94\right)$, interconnected with the red nucleus (MNI -2, $-22,-32)$; and network 9, including the brainstem, posterior insula, bilateral thalamus, and left SMA ( $\mathrm{p}_{\mathrm{FwE}}=0.002$, cluster size $\left.=106\right)$, interconnected with the left SMA (MNI 24, -28, 44). Higher pretherapeutic IC was associated with higher MR volumes, in a network including the anterior default-mode network and bilateral thalamus (ANOVA, $\mathrm{p}_{\mathrm{FWE}}=0.004$, cluster size $=73$ ), interconnected with cerebellar lobule V $(\mathrm{MNI}-12,-70,-22)$. Moreover, in the same network, radiological hyporesponders presented with negative IC values.
\end{abstract}

CONCLUSIONS These findings have clinical implications for predicting MR signature volumes after SRS-T. Here, using

ABBREVIATIONS AC = anterior commissure; $A D L=$ activities of daily living; $B O L D=$ blood oxygenation level-dependent; $D B S=$ deep brain stimulation; $\mathrm{DMN}=$ defaultmode network; DTI = diffusion tensor imaging; ET = essential tremor; FEW = family-wise error; IC = interconnectivity; ICA = independent component analysis; MNI = Montreal Neurological Institute; PC = posterior commissure; rs-fMRI = resting-state functional MRI; SMA = supplementary motor area; SRS = stereotactic radiosurgery; SRS-T = SRS thalamotomy; TSTH = tremor score on the treated hand; Vim = ventral intermediate nucleus.

SUBMITTED May 16, 2018. ACCEPTED July 24, 2018.

INCLUDE WHEN CITING DOI: 10.3171/2018.7.GKS18752.

${ }^{*}$ C.T. and J.R. contributed equally to this work and share first authorship. 
pretherapeutic MRI and data processing without prior hypothesis, the authors showed that pretherapeutic network interconnectivity strength predicts 1-year MR signature volumes following SRS-T.

https://thejns.org/doi/abs/10.3171/2018.7.GKS18752

KEYWORDS stereotactic radiosurgery; Gamma Knife; thalamotomy; Vim; resting-state fMRI

$\mathrm{E}$ SSENTIAL tremor (ET) is the most common movement disorder in adults. ${ }^{24}$ The clinical manifestation of ET is gradual tremor appearance, predominantly in the upper extremities, although the head, voice, legs, and trunk can also be involved..$^{10,11}$ Several hypotheses exist, but the underlying pathophysiology of ET remains unknown. The most common hypothesis presumes that tremor is generated by abnormal oscillations within the motor cortex, the corresponding contralateral motor thalamic area, and the cerebellum, as parts of what is called the "tremor network."5,16,20 Targeting part of this network, in particular at the level of the ventral intermediate nucleus (Vim) of the thalamus, leads to good therapeutic responses. ${ }^{3}$

Drug-resistant ET can benefit from standard stereotactic procedures, including Vim deep brain stimulation $(\mathrm{DBS})^{3}$ and radiofrequency thermocoagulation, ${ }^{18,30}$ with immediate clinical effect. Stereotactic radiosurgery (SRS) aiming at the same target (Vim) is a minimally invasive alternative with three main limitations: indirect targeting, no intraoperative confirmation by electrophysiology, and delayed clinical and radiological effect.,22,25,40 More recently, as an alternative to radiofrequency thermocoagulation, high-intensity focused ultrasound has emerged as a therapeutic option. ${ }^{12,13}$

Resting-state functional MRI (rs-fMRI) is a noninvasive neuroimaging technique that is able to detect fluctuations in blood oxygenation level-dependent (BOLD) signal in the absence of a task, stimulus, or any other explicit attentional demands. ${ }^{17}$ This is based on the assumption that the brain is active in the absence of a task, primarily driven by internal dynamics, with external events modulating rather than determining the activity of the system. ${ }^{17}$ Resting-state fMRI has been successfully used for understanding many pathophysiological conditions, including movement disorders. $^{4,5,16}$ Functional connectivity or interconnectivity (IC) - if within a functional network - has been defined as a measure of temporal dependencies of neuronal activation between anatomically distant brain regions.

In the present study, we attempted to correlate pretherapeutic IC-depicted in rs-fMRI within large-scale distributed neuronal networks - with MR signature volume 1 year after SRS thalamotomy (SRS-T) for ET. This particular aspect could be of potential clinical interest, as recent studies have shown a correlation between this volume and clinical improvement, in the sense that higher volumes are related to better therapeutic responses. ${ }^{8,39}$ Moreover, in a small number of cases, higher MR reactions can be accompanied by edema and/or symptoms, with a need for further corticosteroid therapy. There are no data in the current literature with regard to factors potentially correlating with these MR reactions. Our purpose was to be able to detect, based only on pretherapeutic neuroimaging, particular functional patient profiles that might be charac- teristic for higher MR signature volumes. Our hypothesis was that changes within the previously described "tremor axis" are responsible for a variation in the final MR signature volume, despite standard radiosurgical targeting. We use a methodology implying no prior hypothesis, and so our findings could shed new light on this rather complex radiobiological aspect of SRS-T.

\section{Methods \\ Patients}

Seventeen consecutive patients with ET and 12 healthy controls were included. All had right-sided drug-resistant tremor, treated only with left unilateral SRS-T in Marseille, France, between September 2014 and August 2015. We acquired formal approval from the Ethical Committee (at CHU Timone). All patients gave written informed consent. A neurologist who specialized in movement disorders (T.W.) referred the patients. Patients with bilateral procedures, other types of tremor, previous right SRS-T, epilepsy, ischemic attacks, etc., were excluded. The main indications for SRS-T rather than stereotactic DBS or thalamotomy were medical comorbidities, advanced age, and patient refusal of DBS.

Patient neuroimaging evaluation was performed using a standard protocol and a single 3-T MRI machine (Siemens Skyra). The sequences used included T1-weighted and resting-state fMRI, at both baseline and 1 year after the intervention $(n=17 \times 2)$. A standard clinical protocol at both time points supplemented the previous neuroimaging protocol.

The mean age for patients with ET was 70.1 years (range 49-82 years) and for healthy controls was 69.3 (range 5983 years, $\mathrm{p}>0.05,2$-sample t-test). The mean time to decrease in tremor amplitude was $3.32 \pm 2.7$ months (range 0.5-10 months). The mean duration of symptoms was 38 years (range 6-70 years).

\section{Clinical Evaluation and Outcome Measures}

Every 3 months after SRS-T we (T.W. and J.R.) performed a clinical evaluation. Tremor severity was assessed using the questionnaire designed by Bain et al. ${ }^{2}$ (e.g., activities of daily living [ADL] $)^{2}$ and was also evaluated using the tremor score on the treated hand (TSTH) for the right side, from the Fahn-Tolosa-Marin tremor rating scale $^{14}$ (see Table 1 for demographic data).

\section{SRS-T Procedure}

We first acquired nonstereotactic images, including diffusion tensor imaging (DTI), the day before SRS-T. DTI was further coregistered with the stereotactic sequences. After application of the Leksell coordinate $\mathrm{G}$ frame (Elekta AB), patients underwent both CT and MRI before 
TABLE 1. Demographic data

\begin{tabular}{lcrc}
\hline \multicolumn{1}{c}{ Variable } & Mean & SD & Range \\
\hline Age (yrs) & 70.1 & 9.8 & $49-82$ \\
\hline Duration of symptoms (yrs) & 38 & 19.5 & $6-70$ \\
\hline TSTH & & & \\
\hline$\quad$ Baseline & 18.6 & 5.5 & $8-30$ \\
\hline Decrease in points (1 yr minus baseline) & -11.4 & 4.1 & -4 to -19 \\
\hline ADL & & & \\
\hline Baseline & 29.1 & 12 & $13-49$ \\
\hline$\quad$ Decrease in points (1 yr minus baseline) & -23.06 & 11.9 & 2 to -45 \\
\hline Time to improvement after Vim SRS (mos) & 3.3 & 2.7 & $0.2-10$ \\
\hline
\end{tabular}

SRS. All Vim radiosurgery was performed by same neurosurgeon (J.R.) using the Leksell GammaPlan software (Elekta Instruments AB). On MR ventriculography (T2 constructive interference in steady-state/fast imaging employing steady-state acquisition sequences) we defined the anterior and posterior commissure (AC and PC). We conducted indirect targeting using standard methodology according to Guiot's diagram, placed $2.5 \mathrm{~mm}$ above the AC-PC line and $11 \mathrm{~mm}$ lateral to the wall of the third ventricle. A single 4-mm isocenter was used. The maximal prescription dose was 130 Gy at the $100 \%$ isodose line. We additionally used beam channel blocking to optimize the dose gradient toward the internal capsule. ${ }^{23,35,37,38,40}$

\section{Clinical Characteristics}

The male/female ratio was 5:12. The mean ADL point decrease at 1 year after SRS-T was $-23.06 \pm 11.9$ (range 2 to -45 ) and for TSTH was $-11.4 \pm 4.1$ (range -4 to -19 ; Table 1).

\section{Radiological Answer: MR Signature After Thalamotomy (1 Year After SRS-T)}

The MR signature volume was individually evaluated on T1-weighted Gd contrast-enhanced MRI 1 year after SRS-T, which is considered the definitive radiological answer according to our previously published experiment. ${ }^{39}$ To be sure that we accurately calculated the corresponding volume, each patient's MRI at 1 year after SRS-T was imported into Leksell GammaPlan software. This MRI was coregistered with therapeutic images. Using GammaPlan segmentation tools, we drew the individual volumes and further calculated them. The mean volume was $0.125 \pm$ $0.162 \mathrm{ml}$ (median $0.063 \mathrm{ml}$, range $0.002-0.600 \mathrm{ml}$; Fig. 1). The 90-Gy isodose line, which corresponds to the final MR signature in our experience, ${ }^{29,39}$ was projected to the 1-year result and perfectly matched the MR signature. There was no correlation between thalamotomy volume and ADL decrease $(p>0.05)$, but there was with TSTH decrease $(p=0.01)$.

\section{MRI Data Acquisition}

MRI data acquisition was performed on a head-only Siemens Skyra, a 32-channel, receive-only, phased-array head coil, without contrast (before radiosurgery) and with Gd contrast-enhanced T1-weighted (after radiosurgery)

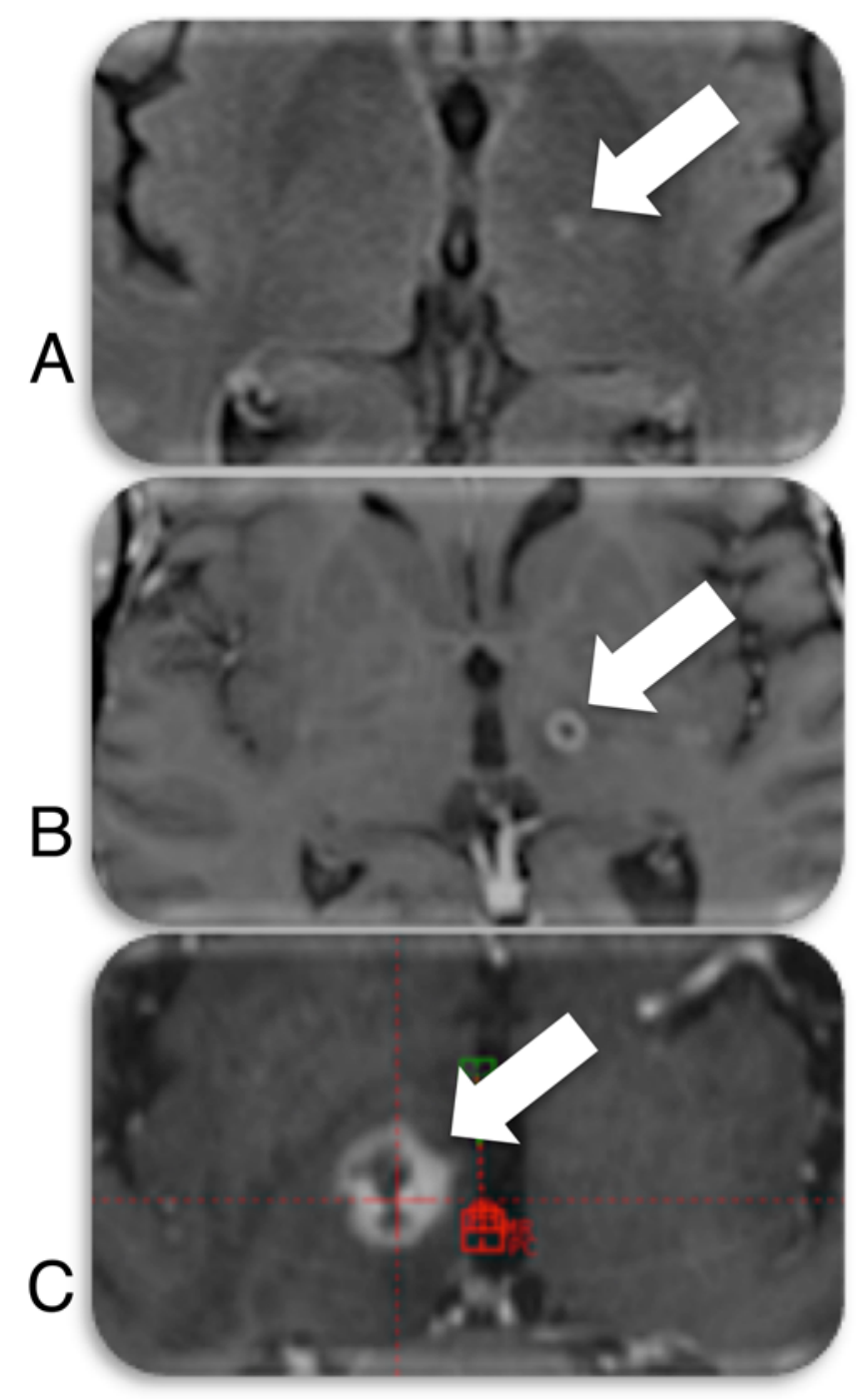

FIG. 1. MR images showing three types of MR signatures (arrows). A: Minimal MRI signature. B: The classic cocade aspect. C: Larger MR signature.

and resting-state fMRI images. The following parameters were used: 1) for T1-weighted imaging, TR/TE 2300/2.98 msec, isotropic voxel $1 \mathrm{~mm}^{3}$, and 160 slices; and 2) for T2*-weighted fast echo planar imaging (BOLD contrast, before the Gd injection), TR/TE $3.3 \mathrm{sec} / 30 \mathrm{msec} / 90^{\circ}$, voxel size $4 \times 4 \times 4 \mathrm{~mm}^{3}, 300$ volumes obtained per subject, and 46 interleaved axial slices. The rs-fMRI experiments, obtained with no explicit task, consisted of a 10-minute run in which participants were asked to relax with their eyes closed, without falling asleep or engaging in tasks. Additionally, a field map was obtained to correct for the effect of field inhomogeneity.

\section{Resting-State fMRI Data Preprocessing and Motion Scrubbing}

The processing of fMRI data was completed using Statistical Parametric Mapping (SPM version 12, http://www. fil.ion.ucl.ac.uk/spm/; Welcome Trust Center for Neuroimaging). Functional scans were realigned to the first scan of the series. Motion is an important potential confound- 
ing factor, especially in patients with movement disorders. Regarding this issue, we computed the standard Power's framewise displacement index for each time point. ${ }^{28}$ When this index exceeded $0.5 \mathrm{~mm}$, the corresponding frame was "scrubbed" along with the one proceeding it and the two following ones (a total of 5 for one time point exceeding the upper limit allowed). We only considered the remaining frames for further analysis. Before radiosurgery, the mean number of frames taken out was 35 (median 15, range $0-135$ ) and at 1 year was also 35 (median 15, range $0-150$ ). Moreover, the images were spatially normalized into the standard anatomical space (e.g., Montreal Neurological Institute coordinates [SPM-MNI]). We also used spatial smoothing using a Gaussian filter and a full width at high maximum of $6 \mathrm{~mm}$. The voxel size generated from the above acquisition parameters was oversampled to $2 \times$ $2 \times 2 \mathrm{~mm}^{3}$. We employed no sedation so as not to interfere with resting-state brain activity by any means.

\section{Resting-State fMRI Network Extraction and Analysis}

Two individuals who were not involved in patient selection, the radiosurgery procedure, or follow-up course examined the resting-state data (C.T. and D.V.D.V.). Grouplevel independent component analysis (ICA) was applied to deconstruct rs-fMRI data into components of temporally coherent spontaneous activity using GIFT ICA Toolbox (http://icatb.sourceforge.net). ${ }^{6}$ The data-driven analytical approach we employed considered only the data of the pretherapeutic cases ( $n=17$, ET patients). We made this choice to limit variability, which might have been induced by also including the healthy controls and the group with the higher MR signature volume 1 year after SRS-T. The total number of components was conventionally fixed at 20. This is a scenario commonly used in the literature for identifying large-scale distributed networks. We verified all maps at the group level and further excluded no component, as all were considered to display neurologically relevant spatial patterns. An overview of these components can be found in our previously published papers. ${ }^{33,36}$ In the present report, we show only the statistically significant networks and their respective interconnected clusters. Using spatial regression, healthy control rs-fMRI data, as well as the 1-year after SRS-T time points, were projected on the respective networks, and the values from the reference cluster extracted.

\section{rs-fMRI Statistical Analysis and Evaluation of Potential Confounders}

Two types of statistical analysis were performed and are further reported in the Results section. We first performed a 2-sample t-test using two groups, at the level of each component: group $1(\mathrm{n}=8)$, with an MR signature volume $<0.063 \mathrm{ml}$ (which was the median MR signature volume 1 year after SRS-T), and group $2(\mathrm{n}=9)$, with an MR signature volume $\geq 0.063 \mathrm{ml}$. These two groups did not significantly differ by age ( $\mathrm{p}=0.16)$, duration of symptoms $(\mathrm{p}=0.13)$, baseline ADL $(\mathrm{p}=0.62)$, ADL point decrease at 1 year $(p=0.32)$, time to tremor arrest $(p=0.65)$, and baseline TSTH $(\mathrm{p}=0.11)$. There was a higher TSTH point decrease at 1 year in patients with larger MR signa- tures (group 2), with a mean of $-13.222 \pm 2.921$, compared with $-9.375 \pm 3.029$ in group $1(\mathrm{p}=0.049)$.

We also performed an ANOVA as implemented in SPM12 (http://www.fil.ion.ucl.ac.uk/spm/software/spm12) as a flexible factorial model, on each component, by using individual subject-level maps and the continuous values of 1-year MR signatures, correlated with baseline IC values. For both analyses, Bonferroni correction was used to address the number of models $(n=20)$. We further reported corrected $p$ values using conventional cluster-level familywise error (FWE) correction.

Stata (version 11, StataCorp LLC) was used to determine the correlation between IC and MR signature values, as well as the previously detailed standard tremor scores.

\section{Results}

\section{Two-Sample t-Test at the Level of Each Network}

Two networks were statistically significant: 1) network 3 , including the brainstem, motor cerebellum, bilateral thalamus, and left supplementary motor area (SMA; $\mathrm{p}_{\mathrm{FWE}}$ $=0.004$, cluster size $=94$ ), interconnected with the red nucleus (MNI -2, -22, -32); and 2) network 4, including the brainstem, posterior insula, bilateral thalamus, and left SMA $\left(\mathrm{p}_{\mathrm{FWE}}=0.002\right.$, cluster size $\left.=106\right)$, interconnected with the left SMA (MNI 24, -28, 44).

For network 3 (Fig. 2A), in the respective cluster, pretherapeutic ET IC was higher than that in healthy controls, and decreased to median values close to those of healthy controls 1 year after SRS-T (Fig. 2B). Furthermore, the median pretherapeutic IC values were negative in group 1 and close to zero in group 2 (Fig. 2C). Thus, higher MR signatures were associated with higher pretherapeutic IC values ( $\mathrm{p}=0.004$, FWE corrected).

For network 4 (Fig. 3A), in patients with ET, differences between 1-year and pretherapeutic IC values (Fig. 3B) correlated with a TSTH decrease in points (Spearman rank correlation coefficient $=0.02$ ). Furthermore, in the respective cluster, pretherapeutic ET IC was zero, while in the healthy controls it was slightly positive, and increased to slightly positive median values close to those in healthy controls 1 year after SRS-T (Fig. 3C). Furthermore, the median pretherapeutic IC values were slightly negative in group 1 and became even more negative 1 year after SRS$\mathrm{T}$; in group 2 they were slightly positive and became even more positive 1 year after SRS-T (Fig. 3D). This network reflects higher and positive pretherapeutic IC values associated with higher MR signature volumes $(\mathrm{p}=0.002$, FWE corrected). In contrast, hyporesponders presented negative pretherapeutic IC values.

\section{MR Signature as a Continuous Value}

One network (number 9) was statistically significant and included IC between parts of the anterior defaultmode network $(\mathrm{DMN})$ and bilateral thalamus $\left(\mathrm{p}_{\mathrm{FWE}}=\right.$ 0.004 , cluster size $=73$ ) with the cerebellar lobule V $(\mathrm{MNI}$ $-12,-70,-22$; Fig. 4A). In the respective cluster, pretherapeutic ET IC was higher and slightly positive compared with healthy controls (negative values), and decreased to median values 1 year after SRS-T (Fig. 4B). Furthermore, higher MR signature volumes were associated with higher 


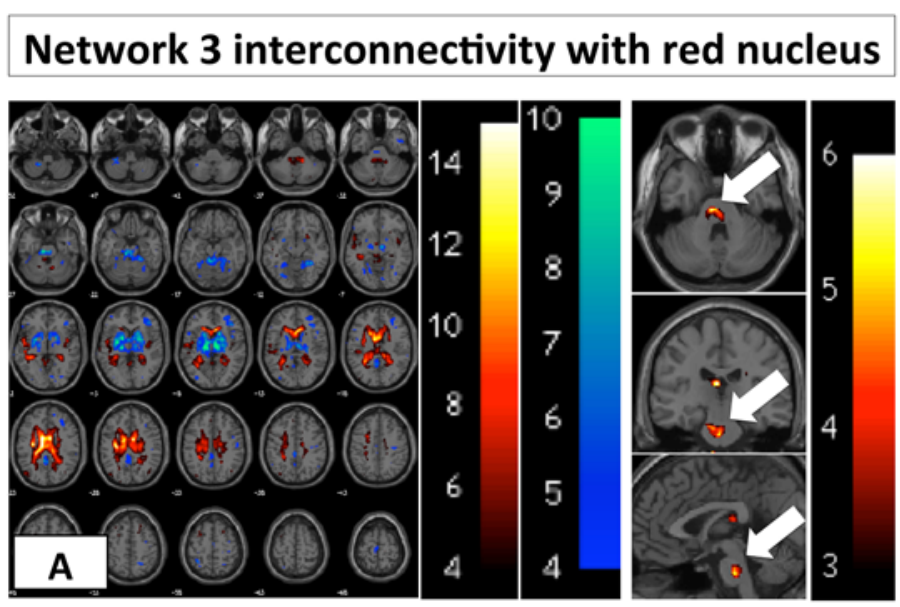

\begin{tabular}{|c|c|c|}
\hline $\begin{array}{c}\text { Healthy controls } \\
(\mathbf{n}=\mathbf{1 2})\end{array}$ & $\begin{array}{c}\text { Pretherapeutic } \\
\text { ET }(\mathbf{n}=\mathbf{1 7})\end{array}$ & $\begin{array}{c}\mathbf{1} \text { year after SRS-T } \\
(\mathbf{n}=\mathbf{1 7})\end{array}$ \\
\hline mean -3.65 & mean -0.69 & mean -3.36 \\
median -3.74 & median -0.87 & median -3.32 \\
range $-9.27->1.15$ & range $-1.41->0.66$ & range $-10.9->5.56$ \\
\hline
\end{tabular}

\begin{tabular}{|c|c|}
\hline $\begin{array}{c}\text { Pretherapeutic ET, } \\
\text { MR signature }<0.063 \mathrm{ml}\end{array}$ & $\begin{array}{c}\text { Pretherapeutic ET, } \\
\text { MR signature }>=0.063 \mathrm{ml}\end{array}$ \\
\hline mean -1.10 & mean -0.33 \\
median -1.06 & median -0.19 \\
range $-1.41->-0.81$ & range $-1.23>0.66$ \\
\hline
\end{tabular}
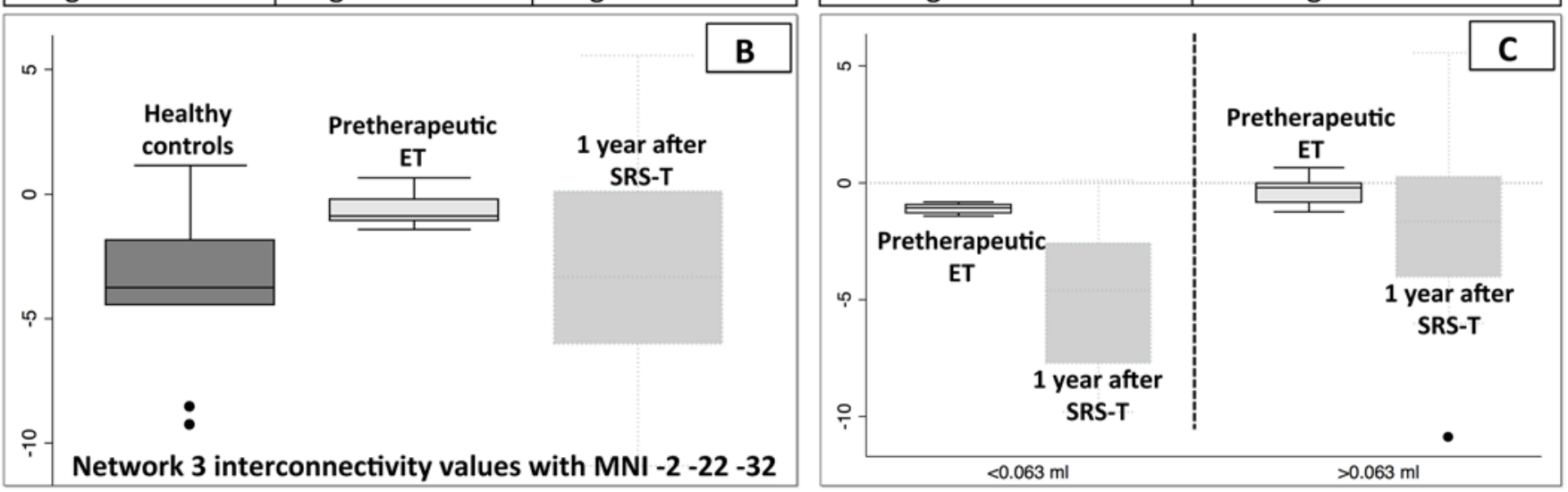

FIG. 2. First statistically significant result of the 2-sample t-test. Network $3(\mathbf{A})$; in the respective cluster, pretherapeutic ET IC was higher than that of healthy controls, and decreased to median values close to those of healthy controls 1 year after SRS-T (B).

Furthermore, the median pretherapeutic IC values were negative in group 1 and close to zero in group 2 (C).

pretherapeutic IC ( $\mathrm{p}=0.004$, FWE corrected; Fig. 4C). In contrast, radiological hyporesponders had negative IC values.

\section{Discussion}

The present study evaluates the possibility of predicting the 1-year MR signature volume after SRS-T, based only on pretherapeutic rs-fMRI, as a noninvasive neuroimaging assessment acquired with minimal patient compliance. Three networks were statistically significant; the core of these networks is the bilateral thalamus. In addition, one could also see the parts of the brainstem, posterior insula, anterior DMN, and left SMA. The interconnected clusters are mainly the red nucleus, left SMA, and motor cerebellum lobule V. The network including the DMN and bilateral thalamus, interconnected with the motor cerebellum lobule $\mathrm{V}$, showed a nice and linear correlation between pretherapeutic IC values and 1-year MR signature volumes. In fact, higher MR signature volumes were correlated with higher pretherapeutic IC values. This finding places the motor cerebellum as a calibrator between the thalamus on one side and the DMN on the other side, in MR signature appearance and volume after SRS-T. Here, we postulate that the cerebellum not only is involved in ET pathophysiology but also plays a major role in the development and volume of the MR reaction after SRS-T.

Aspects of the 1-year MR signature, such as when it appears, its volume, and clinical correlation with regard to SRS-T and the complexity of the radiobiology of SRS, remain fascinating research questions. We postulate that the thalamus most probably plays a central role due to its presence in all the reported networks. A specific and abnormal functional connectivity profile with regard to this targeted anatomical structure might be a key part in understanding the different types of MR signatures. By its multiple functions, acting as a hub, the thalamus is relaying infor- 


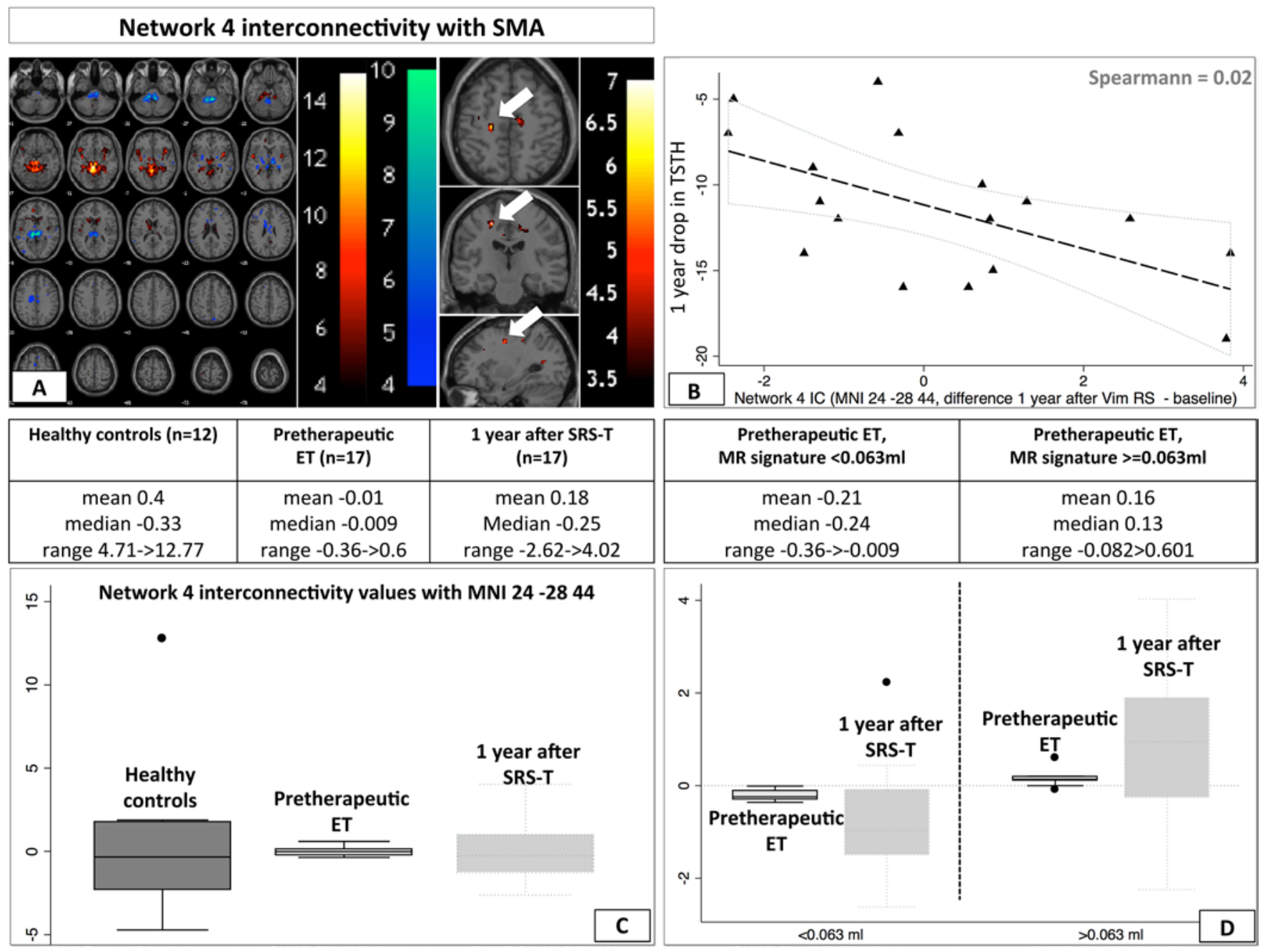

FIG. 3. Second statistically significant result of the 2-sample t-test. Network 4 (A); differences in IC between 1 year and pretherapeutic (B) correlated with the TSTH decrease in points (Spearman rank correlation coefficient $=0.02$ ). Furthermore, in the respective cluster, pretherapeutic ET IC was zero, while in the healthy controls it was slightly positive, and increased to slightly positive median values close to those in healthy controls 1 year after SRS-T (C). Moreover, the median pretherapeutic IC values were slightly negative in group 1 and became even more negative 1 year after SRS-T; in group 2 they were slightly positive and became even more positive 1 year after SRS-T (D).

mation between different subcortical areas and the cerebral cortex, contributing to normal brain homeostasis. The appearance of the anterior insula in another network is not astonishing. In the context of ET, recent studies have specified an increased functional connectivity of sensory motor and salience networks, compared with healthy controls. ${ }^{15}$ Furthermore, in a previous report,${ }^{34}$ we suggested a reorganization of salience networks after SRS-T. We postulated that the insula would act as an integral hub in generating an appropriate behavioral response to salient stimuli. ${ }^{26}$ The present report would additionally confirm a role for this structure in the development of a particular MR signature, while having a specific abnormal functional connectivity profile. Furthermore, we observed parts of the anterior DMN. In a recent report, we suggested that pretherapeutic IC between the anterior DMN and Brodmann's area 10 would be predictive of tremor arrest after SRS-T. ${ }^{33}$ Moreover, in the same report, IC within another network, containing the bilateral thalamus and motor cerebellum lobule $\mathrm{V}$, also found in the present analysis, was predictive of a decrease in tremor amplitude on the treated hand. ${ }^{33}$ Lobule V is mainly associated with motor control. Furthermore, motor and somatosensory representations show largely overlapping activation patterns, with a major cluster focused in lobule V. ${ }^{32}$ With regard to the SMA, it is interconnected with numerous structures by both efferent (including multiple basal ganglia structures such as the putamen, caudate, and ventrolateral and ventral anterior thalamus [targeted in our patients]) and afferent (basal ganglia [ventrolateral and anterior thalamus], insula, midbrain, etc.) connections.

The correlation between 1-year MR signature volume and clinical response has been the subject of constant research in the Marseille group, which recently reviewed 169 cases, with neurological data obtained in 91 of them. ${ }^{8}$ A correlation was established between the volume of MR 


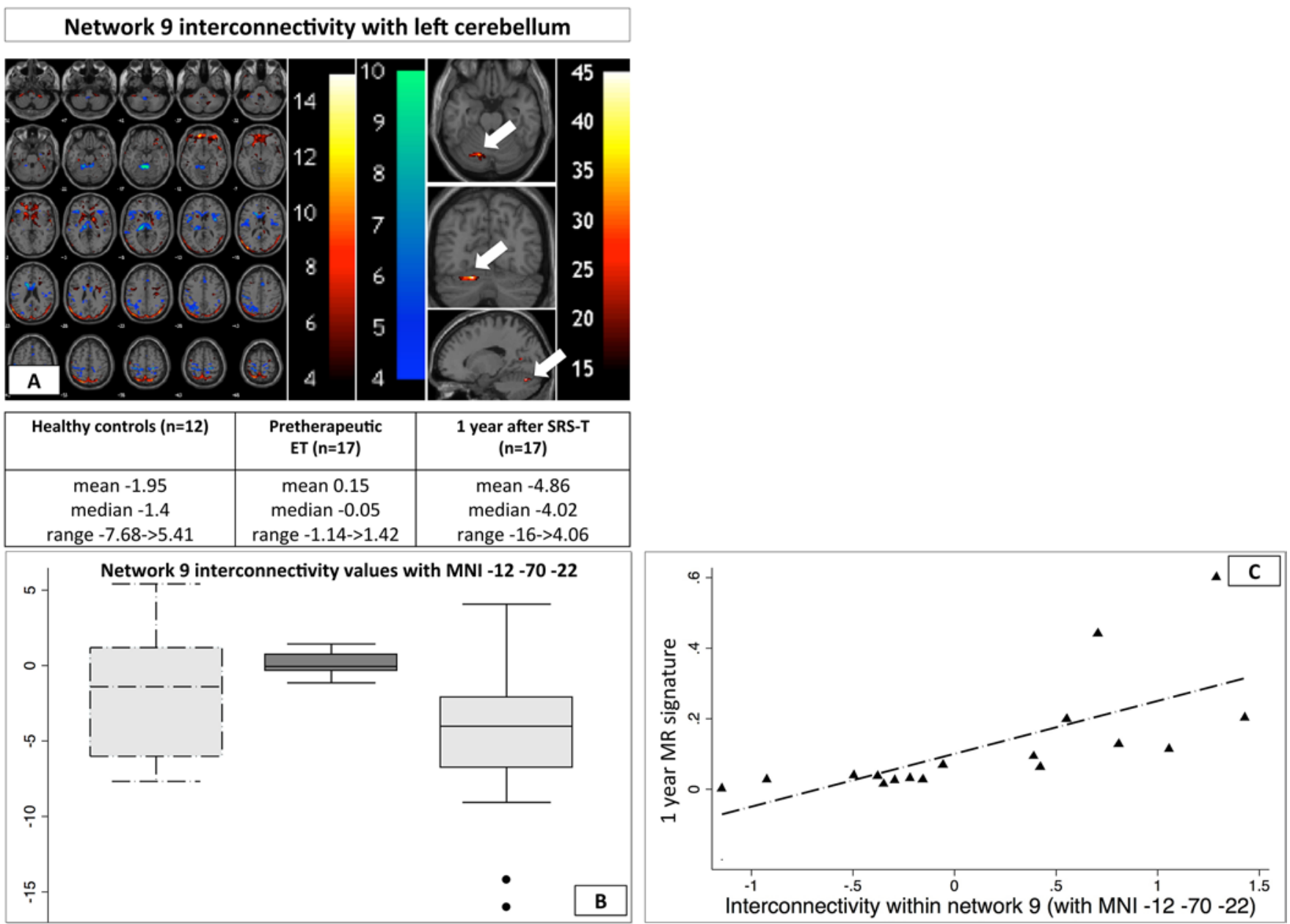

FIG. 4. MR signature taken as a continuous value in an ANOVA model with pretherapeutic IC. Network 9 , including IC between parts of the anterior DMN and bilateral thalamus $\left(p_{\mathrm{FWE}}=0.004\right.$, cluster size $\left.=73\right)$ with the cerebellar lobule $\mathrm{V}(\mathrm{MNI}-12,-70,-22$; A). In the respective cluster, pretherapeutic ET IC was higher and slightly positive compared with that in healthy controls (negative values), and decreased to median values 1 year after SRS-T (B). Furthermore, higher MR signature volumes were associated with higher pretherapeutic IC (C).

signature and percentage of tremor reduction (Pearson's correlation coefficient $=0.26, \mathrm{p}=0.0178$ ). The amount of edema surrounding the lesion was found to be significantly related to clinical improvement $(\mathrm{p}=0.022)$. The "cocade" pattern enhancement type ${ }^{29}$ was strongly related to good outcome $(\mathrm{p}<0.001)$, and the absence of enhancement to the absence of improvement ( $p<0.00001,62 \%$ vs $0.07 \%$ ). We concluded that even though a significant correlation does exist between lesion volume, edema, and clinical improvement, concordance is far from being very strong and linear between the T1-weighted imaging appearance and clinical responses.

During the past three decades, Gamma Knife (GK) thalamotomy has been increasingly used as a minimally invasive, safe, and effective neurosurgical alternative $e^{7,22,40}$ for drug-resistant ET. ${ }^{27}$ Past histological evidence has been mainly limited to very few reports, one of which is the historical series of GK thalamotomy performed by Leksell for intractable pain and further reported by Steiner et al..$^{31}$ The radiation doses used were much higher, compared with what we currently prescribe (e.g., 130 Gy), while the reported error of the target placement as matched to the final result was as low as $1 \mathrm{~mm}$. Furthermore, Leksell's view of the GK was clearly initially intended to mimic the lesional effects of a surgeon's knife, hence the name given to the radiosurgical device. The second histological study, by Kondziolka et al., ${ }^{21}$ evaluated the effect of a 100-Gy radiation dose in the baboon. The authors concluded that radiosurgery of this dose (e.g., lower than in current clinical practice in Vim SRS) generates focal necrosis and axonal degeneration of the thalamus, 6 months after the procedure. More recently, the "cocade theory" 29 has been advocated, which proposes a neuromodulatory effect of Vim SRS, while considering 4 different zones in and around the target: necrotic, subnecrotic, neuromodulation, and no effect.

Our report has numerous possible limitations. The first limitation is the number of subjects. The second limitation could be considered the absence of data in a control group, 1 year after radiosurgery; nevertheless, recent studies have advocated the robustness and reproducibility of functional networks across multiple sessions, including 1 year apart. ${ }^{19}$ A third limitation, which is more relative, is the usage of 
resting-state data, considered by some authors as not directly related to motor performance; however, we aimed to study network changes in the absence of any specific task. A fourth limitation is related to the study design and the assigned time points. In this regard, at which precise moment after SRS-T these changes appear in brain networks is difficult to establish. Moreover, the neurological assessment was not blinded. The anatomical position of some clusters, such as the red nucleus, should be cautiously interpreted due to the relatively low resolution of the fMRI in general.

We chose to use a data-driven approach that has already been widely applied for rs-fMRI to retrieve signatures of major brain networks, as previously demonstrated by other authors. ${ }^{1,9}$ Moreover, we generated these networks using only the data of the pretherapeutic ET cases $(n=17)$ so as to avoid producing any artificial heterogeneity, which could have been induced by either the healthy controls themselves or by the patients with ET at 1 year after SRS$\mathrm{T}$. The projection of their time points on the pretherapeutic time points has further indicated how they differed from those of healthy controls and were altered in patients after this interventional procedure.

\section{Conclusions}

The intimate mechanisms by which some patients develop different types of 1-year MR signatures despite a standard targeting SRS-T procedure remain unknown. We postulate in this study that an abnormal resting-state functional connectivity profile might play a role. The most interesting network is the one with bilateral thalamus and DMN, interconnected with the cerebellum. It has been suggested that the cerebellum plays a role in the pathophysiology of tremor in general and ET in particular. Here, we postulate that it calibrates the thalamo- DMN circuitry and plays a crucial role in higher MR signatures, corresponding to hyperresponders to SRS-T.

\section{Acknowledgments}

We would like to thank all the patients for their willingness to take part in the present study. To Axelle Cretol, for her continuous support and updating of the database on a regular basis; and to Francois Delaire, Marion Vincent, and Jerome Champoudry, for the easy access to all the encoded radiological files. This work has been supported by the Timone University Hospital, the Swiss National Science Foundation (grant no. 205321-157040), the Centre d'Imagerie BioMédicale (CIBM) of the University of Lausanne (UNIL), the University of Geneva (UniGe), the Centre Hospitalier Universitaire Vaudois (CHUV), and the Leenaards and Jeantet Foundations.

\section{References}

1. Afshin-Pour B, Grady C, Strother S: Evaluation of spatiotemporal decomposition techniques for group analysis of fMRI resting state data sets. Neuroimage 87:363-382, 2014

2. Bain PG, Findley LJ, Atchison P, Behari M, Vidailhet M, Gresty M, et al: Assessing tremor severity. J Neurol Neurosurg Psychiatry 56:868-873, 1993

3. Benabid AL, Pollak P, Gao D, Hoffmann D, Limousin P, Gay E, et al: Chronic electrical stimulation of the ventralis intermedius nucleus of the thalamus as a treatment of movement disorders. J Neurosurg 84:203-214, 1996
4. Benito-León J, Louis ED, Romero JP, Hernández-Tamames JA, Manzanedo E, Álvarez-Linera J, et al: Altered functional connectivity in essential tremor: a resting-state fMRI study. Medicine (Baltimore) 94:e1936, 2015

5. Buijink AW, van der Stouwe AM, Broersma M, Sharifi S, Groot PF, Speelman JD, et al: Motor network disruption in essential tremor: a functional and effective connectivity study. Brain 138:2934-2947, 2015

6. Calhoun VD, Adali T, Pearlson GD, Pekar JJ: A method for making group inferences from functional MRI data using independent component analysis. Hum Brain Mapp 14:140151,2001

7. Campbell AM, Glover J, Chiang VL, Gerrard J, Yu JB: Gamma knife stereotactic radiosurgical thalamotomy for intractable tremor: a systematic review of the literature. Radiother Oncol 114:296-301, 2015

8. Carron R, Witjas T, Tancu C, Spatola G, Régis J: Correlations between the clinical results and the MR characteristics of the thalamic lesion in Vim Gammaknife radiosurgery for tremor. J Radiosurg SBRT 5 (1 Suppl):11, 2017 (Abstract)

9. Damoiseaux JS, Rombouts SA, Barkhof F, Scheltens P, Stam CJ, Smith SM, et al: Consistent resting-state networks across healthy subjects. Proc Natl Acad Sci U S A 103:1384813853, 2006

10. Deuschl G, Elble R: Essential tremor-neurodegenerative or nondegenerative disease towards a working definition of ET. Mov Disord 24:2033-2041, 2009

11. Elble RJ: Diagnostic criteria for essential tremor and differential diagnosis. Neurology 54 (11 Suppl 4):S2-S6, 2000

12. Elias WJ, Huss D, Voss T, Loomba J, Khaled M, Zadicario E, et al: A pilot study of focused ultrasound thalamotomy for essential tremor. N Engl J Med 369:640-648, 2013

13. Elias WJ, Lipsman N, Ondo WG, Ghanouni P, Kim YG, Lee W, et al: A randomized trial of focused ultrasound thalamotomy for essential tremor. N Engl J Med 375:730-739, 2016

14. Fahn S, Tolosa E, Marin C: Clinical rating scale for tremor, in Jankovik J, Tolosa E (eds): Parkinson's Disease and Movement Disorders. Baltimore: Urban \& Schwarzenberg, 1988, pp 225-234

15. Fang W, Chen H, Wang H, Zhang H, Liu M, Puneet M, et al: Multiple resting-state networks are associated with tremors and cognitive features in essential tremor. Mov Disord 30:1926-1936, 2015

16. Fang W, Chen H, Wang H, Zhang H, Puneet M, Liu M, et al: Essential tremor is associated with disruption of functional connectivity in the ventral intermediate nucleus-motor cortex - cerebellum circuit. Hum Brain Mapp 37:165-178, 2016

17. Fox MD, Corbetta M, Snyder AZ, Vincent JL, Raichle ME: Spontaneous neuronal activity distinguishes human dorsal and ventral attention systems. Proc Natl Acad Sci U S A 103:10046-10051, 2006

18. Fox MW, Ahlskog JE, Kelly PJ: Stereotactic ventrolateralis thalamotomy for medically refractory tremor in post-levodopa era Parkinson's disease patients. J Neurosurg 75:723730, 1991

19. Guo CC, Kurth F, Zhou J, Mayer EA, Eickhoff SB, Kramer $\mathrm{JH}$, et al: One-year test-retest reliability of intrinsic connectivity network fMRI in older adults. Neuroimage 61:14711483,2012

20. Hua SE, Lenz FA: Posture-related oscillations in human cerebellar thalamus in essential tremor are enabled by voluntary motor circuits. J Neurophysiol 93:117-127, 2005

21. Kondziolka D, Couce M, Niranjan A, Maesawa S, Fellows W: Histology of the 100-Gy thalamotomy in the baboon, in Kondziolka D (ed): Radiosurgery. Basel: Karger, 2002, Vol 4, pp 279-284

22. Kondziolka D, Ong JG, Lee JY, Moore RY, Flickinger JC, Lunsford LD: Gamma Knife thalamotomy for essential tremor. J Neurosurg 108:111-117, 2008 
23. Lefranc M, Carron R, Regis J: Prelemniscal radiations: a new reliable landmark of the thalamic nucleus ventralis intermedius location. Stereotact Funct Neurosurg 93:400-406, 2015

24. Louis ED: Essential tremor. Lancet Neurol 4:100-110, 2005

25. Martínez-Moreno NE, Sahgal A, De Salles A, Hayashi M, Levivier M, Ma L, et al: Stereotactic radiosurgery for tremor: systematic review. J Neurosurg [epub ahead of print February 23, 2018; DOI: 10.3171/2017.8.JNS17749]

26. Menon V, Uddin LQ: Saliency, switching, attention and control: a network model of insula function. Brain Struct Funct 214:655-667, 2010

27. Niranjan A, Raju SS, Kooshkabadi A, Monaco E III, Flickinger JC, Lunsford LD: Stereotactic radiosurgery for essential tremor: retrospective analysis of a 19-year experience. Mov Disord 32:769-777, 2017

28. Power JD, Barnes KA, Snyder AZ, Schlaggar BL, Petersen SE: Spurious but systematic correlations in functional connectivity MRI networks arise from subject motion. Neuroimage 59:2142-2154, 2012

29. Régis J, Carron R, Park M: Is radiosurgery a neuromodulation therapy?: A 2009 Fabrikant award lecture. J Neurooncol 98:155-162, 2010

30. Schuurman PR, Bosch DA, Bossuyt PM, Bonsel GJ, van Someren EJ, de Bie RM, et al: A comparison of continuous thalamic stimulation and thalamotomy for suppression of severe tremor. N Engl J Med 342:461-468, 2000

31. Steiner L, Forster D, Leksell L, Meyerson BA, Boëthius J: Gammathalamotomy in intractable pain. Acta Neurochir (Wien) 52:173-184, 1980

32. Stoodley CJ, Schmahmann JD: Functional topography in the human cerebellum: a meta-analysis of neuroimaging studies. Neuroimage 44:489-501, 2009

33. Tuleasca C, Najdenovska E, Régis J, Witjas T, Girard N, Champoudry J, et al: Pretherapeutic functional neuroimaging predicts tremor arrest after thalamotomy. Acta Neurol Scand 137:500-508, 2018

34. Tuleasca C, Najdenovska E, Regis J, Witjas T, Girard N, Champoudry J, et al: Ventro-lateral motor thalamus abnormal connectivity in essential tremor before and after thalamotomy: a resting-state fMRI study. World Neurosurg 13:e453-e464, 2018

35. Tuleasca C, Pralong E, Najdenovska E, Cuadra MB, Marques JRF, Vingerhoets F, et al: Deep brain stimulation after previous gamma knife thalamotomy of the Vim for essential tremor is feasible! Clinical, electrophysiological and radiological findings. Acta Neurochir (Wien) 159:1371-1373, 2017

36. Tuleasca C, Régis J, Najdenovska E, Witjas T, Girard N, Champoudry J, et al: Pretherapeutic functional imaging allows prediction of head tremor arrest after thalamotomy for essential tremor: the role of altered interconnectivity between thalamolimbic and supplementary motor circuits. World Neurosurg 112:e479-e488, 2018
37. Tuleasca C, Witjas T, Najdenovska E, Verger A, Girard N, Champoudry J, et al: Assessing the clinical outcome of Vim radiosurgery with voxel-based morphometry: visual areas are linked with tremor arrest! Acta Neurochir (Wien) 159:2139-2144, 2017

38. Tuleasca C, Witjas T, Van de Ville D, Najdenovska E, Verger A, Girard N, et al: Right Brodmann area 18 predicts tremor arrest after Vim radiosurgery: a voxel-based morphometry study. Acta Neurochir (Wien) 160:603-609, 2018

39. Witjas T, Carron R, Azulay JP, Regis J: Gammaknife thamamotomy for intractable tremors: clinical outcome and correlations with neuroimaging features. Mov Disord 28 (Suppl 1):1247, 2013 (Abstract)

40. Witjas T, Carron R, Krack P, Eusebio A, Vaugoyeau M, Hariz $\mathrm{M}$, et al: A prospective single-blind study of Gamma Knife thalamotomy for tremor. Neurology 85:1562-1568, 2015

\section{Disclosures}

Dr. Régis reports being a consultant to Medtronic and Elekta. Dr. Girard reports being a consultant to Olea Medical and receiving financial help to attend scientific meetings. Dr. Thiran is a scientific advisor for Intuitive Therapeutics SA.

\section{Author Contributions}

Conception and design: Tuleasca, Van de Ville. Acquisition of data: Régis, Witjas, Girard. Analysis and interpretation of data: Tuleasca, Régis, Najdenovska, Witjas, Van de Ville. Drafting the article: Tuleasca, Van de Ville. Critically revising the article: all authors. Reviewed submitted version of manuscript: all authors. Approved the final version of the manuscript on behalf of all authors: Tuleasca. Statistical analysis: Tuleasca, Faouzi, Van de Ville. Administrative/technical/material support: Tuleasca, Régis, Najdenovska, Witjas, Girard, Delaire, Vincent, Faouzi, Thiran, Bach Cuadra, Levivier, Van de Ville. Study supervision: Régis, Levivier, Van de Ville.

\section{Supplemental Information \\ Previous Presentations}

Parts of this paper were presented in oral form at the 19th Leksell Gamma Knife Society Meeting in Dubai in March 2018.

\section{Additional Affiliations}

Dr. Tuleasca: Department of Neurosurgery, Kremlin-Bicêtre University Hospital, Paris, France.

\section{Correspondence}

Constantin Tuleasca: Centre Hospitalier Universitaire Vaudois, Lausanne, Switzerland. constantin.tuleasca@gmail.com. 